\title{
Deriving New Quasi-Orthogonal Space-Time Block Codes and Relaxed Designing Viewpoints with Full Transmit Diversity
}

\author{
Ming-Yang Chen*, Chiang-Yu Chen ${ }^{\dagger}$, Hua-Chieh Li ${ }^{\ddagger}$, Soo-Chang Pei*, and John M. Cioffi ${ }^{\dagger}$ \\ *Department of Electrical Engineering, National Taiwan University, Taipei, Taiwan 106, R. O. C. \\ Email: roc3.chen@msa.hinet.net, pei@cc.ee.ntu.edu.tw TEL: 886-2-23635251 \# 530 \\ $\dagger^{\dagger}$ Department of Electrical Engineering, Stanford University, CA 94305, U. S. A. \\ Email: Chiangyu.Chen@stanford.edu, cioffi@stanford.edu TEL: 1-650-725-9695 \\ $\ddagger$ Department of Mathematics, National Taiwan Normal University, Taipei, Taiwan 116, R. O. C. \\ Email: li@math.ntnu.edu.tw TEL: 886-2-29320206 \# 219
}

\begin{abstract}
It has been shown that a complex orthogonal design that provides full diversity and full transmission rate is not possible for more than two transmit antennas. This paper presents a new class of quasi-orthogonal space-time block codes using a group-theoretic method. The new designs can achieve full diversity for quadrature phase-shift-keying modulation, like the system using the rotated constellations. Based upon the analysis of the new codes, a relaxed designing viewpoint for full diversity is proposed for arbitrary signal constellations.
\end{abstract}

Keywords-Multi-antenna, space-time block codes, transmit diversity, wireless communications.

\section{INTRODUCTION}

Transmit diversity has played an important role in various areas of multi-antenna systems and the related disciplines. Evidence suggests that space-time block codes (STBC) exploit transmit diversity and reduce decoding complexity significantly (see [8] and those references therein). The resulting bit error rates (BER) are lowered dramatically by the simple transmission schemes. Based upon the idea of orthogonizing transmission matrices, Alamouti [1] first defined the STBC transmission matrix from orthogonal designs for two transmit antennas as

$$
\left(\begin{array}{cc}
z_{1} & z_{2} \\
-z_{2}^{\dagger} & z_{1}^{\dagger}
\end{array}\right)
$$

where $z^{\dagger}$ stands for the complex conjugate of $z$. Later, Tarokh, Jafarkhani, and Calderbank [7] presented a formal setting for orthogonal designs by defining a $T \times N$ unitary matrix with each entry coming from the symbol set $\left\{ \pm z_{1}, \pm z_{1}^{\dagger}, \ldots, \pm z_{K}, \pm z_{K}^{\dagger}\right\}$, where $T, N$, and $K$ represent the block length, the number of transmit antennas, and the number of constellation symbols, respectively. In particular, a design is of full transmission rate (full rate) and minimaldelay if $K=T$ and $T=N$. Due to the unitary properties, orthogonal designs ensure full diversity, and the corresponding maximum-likelihood decoder can be simplified to a linear one.

It is proved in [7] that a complex orthogonal design with minimal-delay cannot achieve full rate for more than two transmit antennas. Therefore, Jafarkhani proposed quasi-orthogonal space-time block codes (QOSTBC) in [3]. A QOSTBC is a

This work was supported by the National Science Council, R.O.C., under Contracts NSC 91-2219-E-002-044 and NSC 93-2752-E-002-006-PAE.
STBC with minimal-delay that divides the columns of the transmission matrix into pairs, where the columns within each pair are not orthogonal but different pairs are orthogonal to each other. In terms of the uncoded BER, the performance of Jafarkhani's QOSTBC shows that the quasi-orthogonal codes perform better than the orthogonal codes in low signal-to-noise ratios (SNR), but are surpassed by the orthogonal codes in high SNRs. This is because QOSTBCs guarantee only half of full-diversity order, and thus the slopes of corresponding BER curves are not as steep as the orthogonal codes. Furthermore, the decoder of these codes works with pairs of transmit symbols instead of a single symbol as in the orthogonal codes. Recently, Sharma and Papadias [5] and Su and Xia [6] presented another scheme called rotation-based method. By computing an angle $\phi$ and rotating the signal constellations, the resulting system may have both full diversity and pairwise maximum-likelihood decoding. Aside from preserving the characters of QOSTBCs in low SNRs, the BER curve also performs a better slope as steep as orthogonal codes. However, the tradeoff is that the size and shape of signal constellations become double and irregular since half of the transmit symbols come from an extra constellation that is obtained from rotating the original one with the phase difference $\phi$.

This paper focuses on a new class of quasi-orthogonal designs representable by a group-theoretic method on generalized quaternion group of order 16. For quadrature phaseshift-keying (QPSK) modulation, these new QOSTBCs can achieve full diversity as the system with a constellation rotation [5] and [6]. Moreover, no extra rotated constellation is needed. Taking advantage of several known results as well as results derived in this paper concerning transmit diversity, we are able to show how relaxed designing viewpoints for full diversity are possible based upon the analysis of our matrices. When compared with [5] and [6], our approach is capable of providing the same BER performance, while only the simplest multi-antenna encoding structure is used.

The rest of the paper is organized as follows. In Section II, some observations between transmission codes and discrete groups are introduced, which contribute the knowledge for constructing our new quasi-orthogonal designs in Section III. Section IV presents the experimental results. A conclusion is given in Section V. 


\section{Relations between Full-Rate Codes And DISCRETE GROUPS}

A complex $\operatorname{STBC} \mathcal{S}\left(z_{1}, \ldots, z_{n}\right)$ of size $n$ is an $n \times n$ matrix with entries the indeterminates $\pm z_{1}, \ldots, \pm z_{n}$, their conjugates $\pm z_{1}^{\dagger}, \ldots, \pm z_{n}^{\dagger}$, or multiples of those indeterminates by $\mathbf{i}$ where $\mathbf{i}^{2}=-1$. Without loss of generality, the first row of $\mathcal{S}\left(z_{1}, \ldots, z_{n}\right)$ is set to $\left(z_{1}, \ldots, z_{n}\right)$. This result is latter generalized to any field $K$ with the conjugation replacing by an automorphism of $K$ and $\mathbf{i}$ replacing by a root of unit in $K$.

Given an STBC $\mathcal{S}\left(z_{1}, \ldots, z_{n}\right)$, let $\mathbf{e}_{i}$ be the row vector $\left(z_{1}, \ldots, z_{n}\right)$ with $z_{i}=1$ and $z_{j}=0$ for $j \neq i$, and $\mathbf{e}_{i}^{\prime}$ be the row vector $\left(z_{1}, \ldots, z_{n}\right)$ with $z_{i}=\mathbf{i}$ and $z_{j}=0$ for $j \neq i$. We have the following results.

Lemma 2.1: If the set

$$
G:=\left\{ \pm \mathcal{S}\left(\mathbf{e}_{1}\right), \ldots, \pm \mathcal{S}\left(\mathbf{e}_{n}\right), \pm \mathcal{S}\left(\mathbf{e}_{1}^{\prime}\right), \ldots, \pm \mathcal{S}\left(\mathbf{e}_{n}^{\prime}\right)\right\}
$$

is a multiplicative group, then

$$
\mathcal{C}:=\left\{\mathcal{S}\left(z_{1}, \ldots, z_{n}\right) \mid z_{1}, \ldots, z_{n} \in \mathbb{C}\right\}
$$

is a ring with identity $\mathcal{S}\left(\mathbf{e}_{1}\right)$ over conventional matrix addition and multiplication, where $\mathbb{C}$ is the field of complex numbers.

Proof: First observe that $\mathcal{C}$ is closed under addition because every entry of $\mathcal{S}\left(z_{1}, \ldots, z_{n}\right)$ is linear. For $\mathcal{S}\left(z_{1}, \ldots, z_{n}\right), \mathcal{S}\left(z_{1}^{\prime}, \ldots, z_{n}^{\prime}\right) \in \mathcal{C}$, it is clear that

$$
\begin{aligned}
& \mathcal{S}\left(z_{1}, \ldots, z_{n}\right) \cdot \mathcal{S}\left(z_{1}^{\prime}, \ldots, z_{n}^{\prime}\right) \\
= & \left(\sum_{i=1}^{n} \operatorname{Re}\left\{z_{i}\right\} \mathcal{S}\left(\mathbf{e}_{i}\right)+\operatorname{Im}\left\{z_{i}\right\} \mathcal{S}\left(\mathbf{e}_{i}^{\prime}\right)\right) \\
& \cdot\left(\sum_{i=1}^{n} \operatorname{Re}\left\{z_{i}^{\prime}\right\} \mathcal{S}\left(\mathbf{e}_{i}\right)+\operatorname{Im}\left\{z_{i}^{\prime}\right\} \mathcal{S}\left(\mathbf{e}_{i}^{\prime}\right)\right) \\
= & \sum_{i, j=1}^{n} \operatorname{Re}\left\{z_{i}\right\} \operatorname{Re}\left\{z_{j}^{\prime}\right\} \mathcal{S}\left(\mathbf{e}_{i}\right) \mathcal{S}\left(\mathbf{e}_{j}\right) \\
& +\sum_{i, j=1}^{n} \operatorname{Re}\left\{z_{i}\right\} \operatorname{Im}\left\{z_{j}^{\prime}\right\} \mathcal{S}\left(\mathbf{e}_{i}\right) \mathcal{S}\left(\mathbf{e}_{j}^{\prime}\right) \\
& +\sum_{i, j=1}^{n} \operatorname{Im}\left\{z_{i}\right\} \operatorname{Re}\left\{z_{j}^{\prime}\right\} \mathcal{S}\left(\mathbf{e}_{i}^{\prime}\right) \mathcal{S}\left(\mathbf{e}_{j}\right) \\
& +\sum_{i, j=1}^{n} \operatorname{Im}\left\{z_{i}\right\} \operatorname{Im}\left\{z_{j}^{\prime}\right\} \mathcal{S}\left(\mathbf{e}_{i}^{\prime}\right) \mathcal{S}\left(\mathbf{e}_{j}^{\prime}\right)
\end{aligned}
$$

is also in $\mathcal{C}$. Therefore, $\mathcal{C}$ is closed under multiplication, that is, for any $M_{1}, M_{2} \in \mathcal{C}$ we have $M_{1} \cdot M_{2} \in \mathcal{C}$. Thus to evaluate $M_{1} \cdot M_{2}$ we only need to know what its first row is. In fact, if the $i$-th row of $M \in \mathcal{C}$ is $\left(\lambda_{1}, \ldots, \lambda_{n}\right)$, then the first row of $\mathcal{S}\left(\mathbf{e}_{i}\right) \cdot M$ is $\left(\lambda_{1}, \ldots, \lambda_{n}\right)$, and thus

$$
\mathcal{S}\left(\mathbf{e}_{i}\right) \cdot M=\mathcal{S}\left(\lambda_{1}, \ldots, \lambda_{n}\right) .
$$

From this, because the first row of $\mathcal{S}\left(\lambda_{1}, \ldots, \lambda_{n}\right)$ is $\left(\lambda_{1}, \ldots, \lambda_{n}\right)$, we get

$$
\mathcal{S}\left(\mathbf{e}_{1}\right) \cdot \mathcal{S}\left(\lambda_{1}, \ldots, \lambda_{n}\right)=\mathcal{S}\left(\lambda_{1}, \ldots, \lambda_{n}\right) .
$$

In other words, $\mathcal{S}\left(\mathbf{e}_{1}\right)$ is the identity matrix and the diagonal entries of $\mathcal{S}\left(z_{1}, \ldots, z_{n}\right)$ are either $z_{1}$ or $z_{1}^{\dagger}$, which means that $\mathcal{S}\left(\mathbf{e}_{1}\right)$ is the identity of both $G$ and $\mathcal{C}$. Combining with the addition and multiplication rules on matrices implies that $\mathcal{C}$ is a ring with identity $\mathcal{S}\left(\mathbf{e}_{1}\right)$.

The following derivation shows an algorithm for deciding the entries of $\mathcal{S}\left(z_{1}, \ldots, z_{n}\right)$ if the multiplication rules of

$$
G=\left\{ \pm \mathcal{S}\left(\mathbf{e}_{1}\right), \ldots, \pm \mathcal{S}\left(\mathbf{e}_{n}\right), \pm \mathcal{S}\left(\mathbf{e}_{1}^{\prime}\right), \ldots, \pm \mathcal{S}\left(\mathbf{e}_{n}^{\prime}\right)\right\}
$$

are given. Suppose that

$$
\mathcal{S}\left(\mathbf{e}_{i}\right) \cdot \mathcal{S}\left(\mathbf{e}_{k}\right)= \pm \mathcal{S}\left(\mathbf{e}_{j}\right) .
$$

We have that the $i$-th row of $\mathcal{S}\left(\mathbf{e}_{k}\right)$ is $\pm \mathbf{e}_{j}$ and hence the $i$-th row of $\mathcal{S}\left(\mathbf{e}_{k}^{\prime}\right)$ is $\pm \mathbf{e}_{j}^{\prime}$. This implies that

$$
\mathcal{S}\left(\mathbf{e}_{i}\right) \cdot \mathcal{S}\left(\mathbf{e}_{k}^{\prime}\right)= \pm \mathcal{S}\left(\mathbf{e}_{j}^{\prime}\right) .
$$

In other words, the $(i, j)$-th entry of $\mathcal{S}\left(z_{1}, \ldots, z_{n}\right)$ is $\pm z_{k}$ or $\pm z_{k}^{\dagger}$. More precisely, the $(i, j)$-th entry of $\mathcal{S}\left(z_{1}, \ldots, z_{n}\right)$ corresponding to the following four cases

$$
\begin{aligned}
& (a)\left\{\begin{array}{l}
\mathcal{S}\left(\mathbf{e}_{i}\right) \cdot \mathcal{S}\left(\mathbf{e}_{k}\right)=\mathcal{S}\left(\mathbf{e}_{j}\right) \\
\mathcal{S}\left(\mathbf{e}_{i}\right) \cdot \mathcal{S}\left(\mathbf{e}_{k}^{\prime}\right)=\mathcal{S}\left(\mathbf{e}_{j}^{\prime}\right)
\end{array}\right. \\
& \left(\text { b) } \left\{\begin{array}{l}
\mathcal{S}\left(\mathbf{e}_{i}\right) \cdot \mathcal{S}\left(\mathbf{e}_{k}\right)=-\mathcal{S}\left(\mathbf{e}_{j}\right) \\
\mathcal{S}\left(\mathbf{e}_{i}\right) \cdot \mathcal{S}\left(\mathbf{e}_{k}^{\prime}\right)=-\mathcal{S}\left(\mathbf{e}_{j}^{\prime}\right)
\end{array}\right.\right. \\
& \left(\text { c) } \left\{\begin{array}{l}
\mathcal{S}\left(\mathbf{e}_{i}\right) \cdot \mathcal{S}\left(\mathbf{e}_{k}\right)=\mathcal{S}\left(\mathbf{e}_{j}\right) \\
\mathcal{S}\left(\mathbf{e}_{i}\right) \cdot \mathcal{S}\left(\mathbf{e}_{k}^{\prime}\right)=-\mathcal{S}\left(\mathbf{e}_{j}^{\prime}\right)
\end{array}\right.\right. \\
& \text { (d) }\left\{\begin{array}{l}
\mathcal{S}\left(\mathbf{e}_{i}\right) \cdot \mathcal{S}\left(\mathbf{e}_{k}\right)=-\mathcal{S}\left(\mathbf{e}_{j}\right) \\
\mathcal{S}\left(\mathbf{e}_{i}\right) \cdot \mathcal{S}\left(\mathbf{e}_{k}^{\prime}\right)=\mathcal{S}\left(\mathbf{e}_{j}^{\prime}\right)
\end{array}\right.
\end{aligned}
$$

are $(a): z_{k},(b):-z_{k},(c): z_{k}^{\dagger},(d):-z_{k}^{\dagger}$, respectively. Analogously, if

$$
\mathcal{S}\left(\mathbf{e}_{i}\right) \cdot \mathcal{S}\left(\mathbf{e}_{k}\right)= \pm \mathcal{S}\left(\mathbf{e}_{j}^{\prime}\right),
$$

then the $i$-th row of $\mathcal{S}\left(\mathbf{e}_{k}\right)$ is $\pm \mathbf{e}_{j}^{\prime}$. Thus the $i$-th row of $\mathcal{S}\left(\mathbf{e}_{k}^{\prime}\right)$ is $\pm \mathbf{e}_{j}$ which leads to

$$
\mathcal{S}\left(\mathbf{e}_{i}\right) \cdot \mathcal{S}\left(\mathbf{e}_{k}^{\prime}\right)= \pm \mathcal{S}\left(\mathbf{e}_{j}\right) .
$$

The $(i, j)$-th entry of $\mathcal{S}\left(z_{1}, \ldots, z_{n}\right)$ corresponding to the following four cases

$$
\begin{aligned}
& (e)\left\{\begin{array}{l}
\mathcal{S}\left(\mathbf{e}_{i}\right) \cdot \mathcal{S}\left(\mathbf{e}_{k}\right)=\mathcal{S}\left(\mathbf{e}_{j}^{\prime}\right) \\
\mathcal{S}\left(\mathbf{e}_{i}\right) \cdot \mathcal{S}\left(\mathbf{e}_{k}^{\prime}\right)=-\mathcal{S}\left(\mathbf{e}_{j}\right)
\end{array}\right. \\
& (f)\left\{\begin{array}{l}
\mathcal{S}\left(\mathbf{e}_{i}\right) \cdot \mathcal{S}\left(\mathbf{e}_{k}\right)=-\mathcal{S}\left(\mathbf{e}_{j}^{\prime}\right) \\
\mathcal{S}\left(\mathbf{e}_{i}\right) \cdot \mathcal{S}\left(\mathbf{e}_{k}^{\prime}\right)=\mathcal{S}\left(\mathbf{e}_{j}\right)
\end{array}\right. \\
& (g)\left\{\begin{array}{l}
\mathcal{S}\left(\mathbf{e}_{i}\right) \cdot \mathcal{S}\left(\mathbf{e}_{k}\right)=\mathcal{S}\left(\mathbf{e}_{j}^{\prime}\right) \\
\mathcal{S}\left(\mathbf{e}_{i}\right) \cdot \mathcal{S}\left(\mathbf{e}_{k}^{\prime}\right)=\mathcal{S}\left(\mathbf{e}_{j}\right)
\end{array}\right. \\
& (h)\left\{\begin{array}{l}
\mathcal{S}\left(\mathbf{e}_{i}\right) \cdot \mathcal{S}\left(\mathbf{e}_{k}\right)=-\mathcal{S}\left(\mathbf{e}_{j}^{\prime}\right) \\
\mathcal{S}\left(\mathbf{e}_{i}\right) \cdot \mathcal{S}\left(\mathbf{e}_{k}^{\prime}\right)=-\mathcal{S}\left(\mathbf{e}_{j}\right)
\end{array}\right.
\end{aligned}
$$

are $(e): \mathbf{i} z_{k},(f):-\mathbf{i} z_{k},(g): \mathbf{i} z_{k}^{\dagger},(h):-\mathbf{i} z_{k}^{\dagger}$, respectively. 


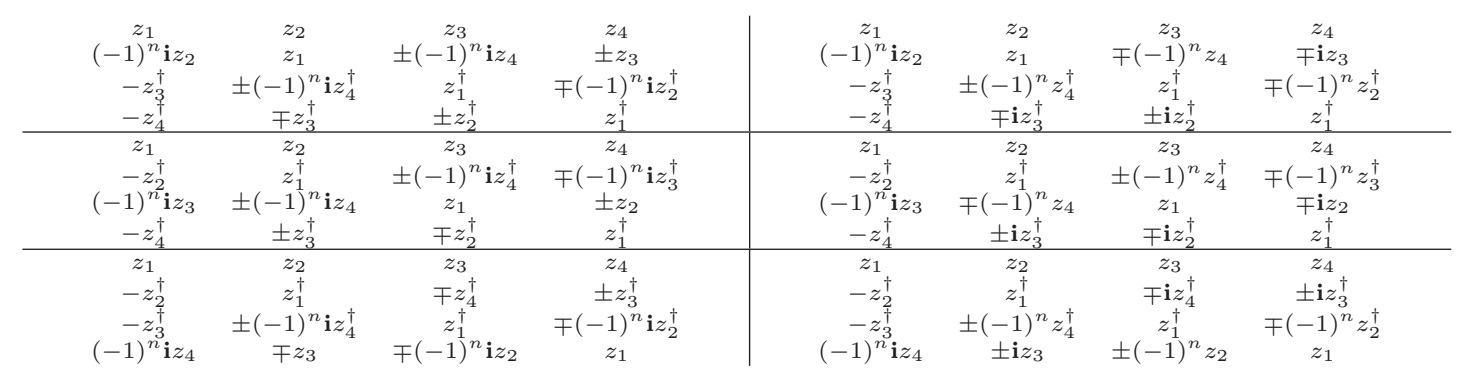

TABLE I

THE $4 \times 4$ QUASI-ORTHOGONAL SPACE-TIME BLOCK CODES.

\section{NEW QUASI-ORTHOGONAL DESIGNS FROM GENERALIZED QUATERNION GROUP}

This section presents new quasi-orthogonal space-time block codes by applying the results $(a) \sim(h)$ discussed in Section II on $Q_{16}$, the generalized quaternion group of order 16 [2]. The group $Q_{16}$ is generated by two elements $a$ and $b$ where $a$ is of order 8 and $b$ is of order 4 with the relation

$$
a^{4}=b^{2} \quad \text { and } \quad a \cdot b=b \cdot a^{-1} .
$$

Explicitly, we can write $Q_{16}$ as

$$
\left\{1, a, a^{2}, a^{3}, a^{4}, a^{5}, a^{6}, a^{7}, b, a b, a^{2} b, a^{3} b, a^{4} b, a^{5} b, a^{6} b, a^{7} b\right\} .
$$

In $Q_{16}, a^{4}$ is the only element of order 2 and $a, a^{3}, a^{5}, a^{7}$ are those elements of order 8 . All the other elements are of order 4 except the identity. Moreover, there is only one cyclic normal subgroup of order 4 in $Q_{16}$ which is generated by $a^{2}$. Before illustrating all the permutations between $G$ and $Q_{16}$, we first give the following Lemma 3.1 and show the Example 3.2 later for clearance.

\section{Lemma 3.1: If}

$$
G=\left\{ \pm \mathcal{S}\left(\mathbf{e}_{1}\right), \ldots, \pm \mathcal{S}\left(\mathbf{e}_{n}\right), \pm \mathcal{S}\left(\mathbf{e}_{1}^{\prime}\right), \ldots, \pm \mathcal{S}\left(\mathbf{e}_{n}^{\prime}\right)\right\}
$$

is a multiplicative group, then

$$
\mathcal{N}:=\left\{\mathcal{S}\left(\mathbf{e}_{1}\right), \mathcal{S}\left(\mathbf{e}_{1}^{\prime}\right),-\mathcal{S}\left(\mathbf{e}_{1}\right),-\mathcal{S}\left(\mathbf{e}_{1}^{\prime}\right)\right\}
$$

is a cyclic normal subgroup of $G$.

Proof: Recall that $\mathcal{S}\left(\mathbf{e}_{1}\right)$ is the identity matrix and the diagonal entries of $\mathcal{S}\left(z_{1}, \ldots, z_{n}\right)$ are either $z_{1}$ or $z_{1}^{\dagger}$. By $\mathcal{S}\left(\mathbf{e}_{1}^{\prime}\right)=\mathcal{S}\left(\mathbf{i e}_{1}\right), \mathcal{S}\left(\mathbf{e}_{1}^{\prime}\right)$ is a diagonal matrix with $(i, i)$ th entries being $\pm \mathbf{i}$ for $i=1, \ldots, n$. Hence it is clear that $\mathcal{S}\left(\mathbf{e}_{1}^{\prime}\right)^{2}=-\mathcal{S}\left(\mathbf{e}_{1}\right), \mathcal{S}\left(\mathbf{e}_{1}^{\prime}\right)^{3}=-\mathcal{S}\left(\mathbf{e}_{1}^{\prime}\right)$, and $\mathcal{S}\left(\mathbf{e}_{1}^{\prime}\right)^{4}=$ $\mathcal{S}\left(\mathbf{e}_{1}\right)$, which means that $N$ is a cyclic subgroup of $G$. Therefore it suffices to show that $N$ is normal. Since the $i$-th row of $\mathcal{S}\left(\mathbf{e}_{1}^{\prime}\right)$ is $\pm \mathbf{e}_{i}^{\prime}$, we may obtain

$$
\mathcal{S}\left(\mathbf{e}_{i}\right) \cdot \mathcal{S}\left(\mathbf{e}_{1}^{\prime}\right)= \pm \mathcal{S}\left(\mathbf{e}_{i}^{\prime}\right) .
$$

By using the similar argument as in (1), it boils down that

$$
\mathcal{S}\left(\mathbf{e}_{i}^{\prime}\right) \cdot M=\mathcal{S}\left(\mathbf{i} \lambda_{1}, \ldots, \mathbf{i} \lambda_{n}\right)
$$

where $\left(\lambda_{1}, \ldots, \lambda_{n}\right)$ is the $i$-th row of $M$. In particular,

$$
\mathcal{S}\left(\mathbf{e}_{1}^{\prime}\right) \cdot \mathcal{S}\left(\mathbf{e}_{i}\right)=\mathcal{S}\left(\mathbf{i e}_{i}\right)=\mathcal{S}\left(\mathbf{e}_{i}^{\prime}\right) .
$$

Thus combining (2) and (3) gives us that

$$
\mathcal{S}\left(\mathbf{e}_{i}\right) \cdot \mathcal{S}\left(\mathbf{e}_{1}^{\prime}\right) \cdot \mathcal{S}\left(\mathbf{e}_{i}\right)^{-1}= \pm \mathcal{S}\left(\mathbf{e}_{1}^{\prime}\right) \in N,
$$

for all $i \in\{1, \ldots, n\}$. Consequently,

$\mathcal{S}\left(\mathbf{e}_{i}^{\prime}\right) \cdot \mathcal{S}\left(\mathbf{e}_{1}^{\prime}\right) \cdot \mathcal{S}\left(\mathbf{e}_{i}^{\prime}\right)^{-1}=\mathcal{S}\left(\mathbf{e}_{1}^{\prime}\right) \cdot\left( \pm \mathcal{S}\left(\mathbf{e}_{1}^{\prime}\right)\right) \cdot \mathcal{S}\left(\mathbf{e}_{1}^{\prime}\right)^{-1} \in N$,

for all $i \in\{1, \ldots, n\}$. This implies that $N$ is normal.

Therefore in the case that $G \cong Q_{16}{ }^{\ddagger}$, we have

$$
\left\{\mathcal{S}\left(\mathbf{e}_{1}\right), \mathcal{S}\left(\mathbf{e}_{1}^{\prime}\right),-\mathcal{S}\left(\mathbf{e}_{1}\right),-\mathcal{S}\left(\mathbf{e}_{1}^{\prime}\right)\right\} \cong\left\{1, a^{2}, a^{4}, a^{6}\right\}
$$

by Lemma 3.1. Note that $\mathcal{S}\left(\mathbf{e}_{1}\right)$ is the identity and $-\mathcal{S}\left(\mathbf{e}_{1}\right)$ is of order 2 in $G$. Suppose that $\mathcal{S}\left(\mathbf{e}_{2}\right)$ is an element of order 8 in $G$. Then

$$
\mathcal{S}\left(\mathbf{e}_{2}\right)^{2}= \pm \mathcal{S}\left(\mathbf{e}_{1}^{\prime}\right),
$$

hence $\pm \mathcal{S}\left(\mathbf{e}_{2}\right)$ and $\pm \mathcal{S}\left(\mathbf{e}_{2}^{\prime}\right)$ are all the elements of $G$ which have order 8 . This implies that

$$
\pm \mathcal{S}\left(\mathbf{e}_{3}\right), \pm \mathcal{S}\left(\mathbf{e}_{3}^{\prime}\right), \pm \mathcal{S}\left(\mathbf{e}_{4}\right), \pm \mathcal{S}\left(\mathbf{e}_{4}^{\prime}\right)
$$

are all elements in $G$ of order 4 and thus

$$
\mathcal{S}\left(\mathbf{e}_{3}\right)^{2}=\mathcal{S}\left(\mathbf{e}_{3}^{\prime}\right)^{2}=\mathcal{S}\left(\mathbf{e}_{4}\right)^{2}=\mathcal{S}\left(\mathbf{e}_{4}^{\prime}\right)^{2}=-\mathcal{S}\left(\mathbf{e}_{1}\right) .
$$

Now consider the product $\mathcal{S}\left(\mathbf{e}_{2}\right) \cdot \mathcal{S}\left(\mathbf{e}_{3}\right)$. If

$$
\mathcal{S}\left(\mathbf{e}_{2}\right) \cdot \mathcal{S}\left(\mathbf{e}_{3}\right) \in\left\{ \pm \mathcal{S}\left(\mathbf{e}_{2}\right), \pm \mathcal{S}\left(\mathbf{e}_{2}^{\prime}\right), \pm \mathcal{S}\left(\mathbf{e}_{3}\right), \pm \mathcal{S}\left(\mathbf{e}_{3}^{\prime}\right)\right\},
$$

a contradiction likes

$$
\mathcal{S}\left(\mathbf{e}_{2}\right) \text { or } \mathcal{S}\left(\mathbf{e}_{3}\right) \in\left\{ \pm \mathcal{S}\left(\mathbf{e}_{1}\right), \pm \mathcal{S}\left(\mathbf{e}_{1}^{\prime}\right)\right\}
$$

would happen from (4) and (5). Similarly, if

$$
\mathcal{S}\left(\mathbf{e}_{2}\right) \cdot \mathcal{S}\left(\mathbf{e}_{3}\right) \in\left\{ \pm \mathcal{S}\left(\mathbf{e}_{1}\right), \pm \mathcal{S}\left(\mathbf{e}_{1}^{\prime}\right)\right\},
$$

by (5) a contradiction

$$
\mathcal{S}\left(\mathbf{e}_{2}\right) \in\left\{ \pm \mathcal{S}\left(\mathbf{e}_{3}\right), \pm \mathcal{S}\left(\mathbf{e}_{3}^{\prime}\right)\right\},
$$

\footnotetext{
¥The notation $G_{1} \cong G_{2}$ is used to denote that there exists a group isomorphism between $G_{1}$ and $G_{2}$.
} 
would be gotten, too. Therefore, we have

$$
\mathcal{S}\left(\mathbf{e}_{2}\right) \cdot \mathcal{S}\left(\mathbf{e}_{3}\right) \in\left\{ \pm \mathcal{S}\left(\mathbf{e}_{4}\right), \pm \mathcal{S}\left(\mathbf{e}_{4}^{\prime}\right)\right\} .
$$

Combining (3), (4), (5), and (6), the multiplication rules of $G$ can be completely determined. Using the consequences $(a) \sim(h)$ derived in Section II, we can construct exactly eight STBCs in the case that $\mathcal{S}\left(\mathbf{e}_{2}\right)$ has order 8. Applying similar argument to the case that $\mathcal{S}\left(\mathbf{e}_{3}\right)$ and $\mathcal{S}\left(\mathbf{e}_{4}\right)$ is an element of order 8 , respectively, there are totally 24 STBCs of size 4 which represent $Q_{16}$. The complete list is written down in Table I, and it is clear that those STBCs are really quasiorthogonal.

Example 3.2: Suppose that $\mathcal{S}\left(\mathbf{e}_{2}\right)^{2}=\mathcal{S}\left(\mathbf{e}_{1}^{\prime}\right)$ and $\mathcal{S}\left(\mathbf{e}_{2}\right)$. $\mathcal{S}\left(\mathbf{e}_{3}\right)=\mathcal{S}\left(\mathbf{e}_{4}\right)$ in (4) and (6), respectively. Combining with (3) and (5), we obtain the multiplication rules of $G$ as the following tables.

\begin{tabular}{|c|c|c|c|c|}
\hline$\cdot$ & $\mathcal{S}\left(\mathbf{e}_{1}\right)$ & $\mathcal{S}\left(\mathbf{e}_{2}\right)$ & $\mathcal{S}\left(\mathbf{e}_{3}\right)$ & $\mathcal{S}\left(\mathbf{e}_{4}\right)$ \\
\hline $\mathcal{S}\left(\mathbf{e}_{1}\right)$ & $\mathcal{S}\left(\mathbf{e}_{1}\right)$ & $\mathcal{S}\left(\mathbf{e}_{2}\right)$ & $\mathcal{S}\left(\mathbf{e}_{3}\right)$ & $\mathcal{S}\left(\mathbf{e}_{4}\right)$ \\
\hline $\mathcal{S}\left(\mathbf{e}_{2}\right)$ & $\mathcal{S}\left(\mathbf{e}_{2}\right)$ & $\mathcal{S}\left(\mathbf{e}_{1}^{\prime}\right)$ & $-\mathcal{S}\left(\mathbf{e}_{4}^{\prime}\right)$ & $\mathcal{S}\left(\mathbf{e}_{3}\right)$ \\
\hline $\mathcal{S}\left(\mathbf{e}_{3}\right)$ & $\mathcal{S}\left(\mathbf{e}_{3}\right)$ & $\mathcal{S}\left(\mathbf{e}_{4}\right)$ & $-\mathcal{S}\left(\mathbf{e}_{1}\right)$ & $-\mathcal{S}\left(\mathbf{e}_{2}\right)$ \\
\hline $\mathcal{S}\left(\mathbf{e}_{4}\right)$ & $\mathcal{S}\left(\mathbf{e}_{4}\right)$ & $\mathcal{S}\left(\mathbf{e}_{3}^{\prime}\right)$ & $\mathcal{S}\left(\mathbf{e}_{2}^{\prime}\right)$ & $-\mathcal{S}\left(\mathbf{e}_{1}\right)$ \\
\hline \hline$\cdot$ & $\mathcal{S}\left(\mathbf{e}_{1}\right)$ & $\mathcal{S}\left(\mathbf{e}_{2}\right)$ & $\mathcal{S}\left(\mathbf{e}_{3}\right)$ & $\mathcal{S}\left(\mathbf{e}_{4}\right)$ \\
\hline $\mathcal{S}\left(\mathbf{e}_{1}^{\prime}\right)$ & $\mathcal{S}\left(\mathbf{e}_{1}^{\prime}\right)$ & $\mathcal{S}\left(\mathbf{e}_{2}^{\prime}\right)$ & $-\mathcal{S}\left(\mathbf{e}_{3}^{\prime}\right)$ & $-\mathcal{S}\left(\mathbf{e}_{4}^{\prime}\right)$ \\
\hline $\mathcal{S}\left(\mathbf{e}_{2}^{\prime}\right)$ & $\mathcal{S}\left(\mathbf{e}_{2}^{\prime}\right)$ & $-\mathcal{S}\left(\mathbf{e}_{1}\right)$ & $-\mathcal{S}\left(\mathbf{e}_{4}\right)$ & $-\mathcal{S}\left(\mathbf{e}_{3}^{\prime}\right)$ \\
\hline $\mathcal{S}\left(\mathbf{e}_{3}^{\prime}\right)$ & $\mathcal{S}\left(\mathbf{e}_{3}^{\prime}\right)$ & $\mathcal{S}\left(\mathbf{e}_{4}^{\prime}\right)$ & $\mathcal{S}\left(\mathbf{e}_{1}^{\prime}\right)$ & $\mathcal{S}\left(\mathbf{e}_{2}^{\prime}\right)$ \\
\hline $\mathcal{S}\left(\mathbf{e}_{4}^{\prime}\right)$ & $\mathcal{S}\left(\mathbf{e}_{4}^{\prime}\right)$ & $-\mathcal{S}\left(\mathbf{e}_{3}\right)$ & $\mathcal{S}\left(\mathbf{e}_{2}\right)$ & $\mathcal{S}\left(\mathbf{e}_{1}^{\prime}\right)$ \\
\hline
\end{tabular}

In particular, $\mathcal{S}\left(\mathbf{e}_{3}\right) \cdot \mathcal{S}\left(\mathbf{e}_{2}\right)$ can be obtained through

$$
\begin{aligned}
\mathcal{S}\left(\mathbf{e}_{3}\right) \cdot \mathcal{S}\left(\mathbf{e}_{2}\right) & =-\mathcal{S}\left(\mathbf{e}_{1}^{\prime}\right) \cdot \mathcal{S}\left(\mathbf{e}_{1}^{\prime}\right) \cdot \mathcal{S}\left(\mathbf{e}_{3}\right) \cdot \mathcal{S}\left(\mathbf{e}_{2}\right) \\
& =-\mathcal{S}\left(\mathbf{e}_{1}^{\prime}\right) \cdot \mathcal{S}\left(\mathbf{e}_{3}^{\prime}\right) \cdot \mathcal{S}\left(\mathbf{e}_{2}\right) \\
& =-\mathcal{S}\left(\mathbf{e}_{1}^{\prime}\right) \cdot \mathcal{S}\left(\mathbf{e}_{2}\right) \cdot \mathcal{S}\left(\mathbf{e}_{4}\right) \cdot \mathcal{S}\left(\mathbf{e}_{2}\right) \\
& =-\mathcal{S}\left(\mathbf{e}_{1}^{\prime}\right) \cdot \mathcal{S}\left(\mathbf{e}_{4}\right) \\
& =-\mathcal{S}\left(\mathbf{e}_{4}^{\prime}\right) .
\end{aligned}
$$

From $(a) \sim(h)$ in Section II, the corresponding QOSTBC is

$$
\left(\begin{array}{cccc}
z_{1} & z_{2} & z_{3} & z_{4} \\
\mathbf{i} z_{2} & z_{1} & \mathbf{i} z_{4} & z_{3} \\
-z_{3}^{\dagger} & \mathbf{i} z_{4}^{\dagger} & z_{1}^{\dagger} & -\mathbf{i} z_{2}^{\dagger} \\
-z_{4}^{\dagger} & -z_{3}^{\dagger} & z_{2}^{\dagger} & z_{1}^{\dagger}
\end{array}\right)
$$

\section{Simulation Results and Designing Viewpoints}

In Fig. 1, the BER performance of our new codes is presented at $2 \mathrm{bits} / \mathrm{s} / \mathrm{Hz}$. The simulation results of three other transmission schemes are also shown. The simulation setup is the same as the one used in [3], which uses QPSK constellation for QOSTBCs and uncoded scheme, and uses 16-quadrature amplitude modulation (QAM) for the rate- $\frac{1}{2}$ orthogonal STBC while the decoding is done by maximum-likelihood (ML) decoding. According to our simulation results, all the 24 codes perform equally well. Hence, only the SNR-BER curve for (7) is shown to represent the new codes. It is known that in general, QOSTBCs can achieve only half of full diversity.
Thus, the slopes of corresponding SNR-BER curves should be less than the slopes of orthogonal STBCs, which implies that orthogonal codes have better performance in high SNRs. On the other hand, when the SNR is low, full-rate QOSTBCs have lower BER than non-full-rate STBCs because of their higher transmission efficiency. In Fig 1, it can be seen that the QOSTBC proposed by Jafarkhani obeys the reasoning. However, surprisingly, as can be observed from the figure, the new code performs better than Jafarkhani's QOSTBC, especially in high SNRs. Moreover, the code shows consistent $3 \mathrm{~dB}$ gain over the rate- $\frac{1}{2}$ orthogonal STBC. This also implies that the new code achieves full diversity order as orthogonal designs do.

Further analysis of the difference matrices for these codes explains the phenomenon more clearly, where a difference matrix is defined as

$$
\mathcal{S}\left(z_{1}, \ldots, z_{n}\right)-\mathcal{S}\left(z_{1}^{\prime}, \ldots, z_{n}^{\prime}\right),
$$

with $z_{1} \ldots, z_{n}, z_{1}^{\prime}, \ldots, z_{n}^{\prime}$ being complex numbers in the given constellations and not all $z_{i}$ 's are equal to $z_{i}^{\prime}$ 's. Since it is known in [4] that the rank of a difference matrix for any $N=$ $T=K=4$ QOSTBC is either 2 or 4 . In Table II, only the numbers of rank 2 and rank 4 difference matrices are shown. According to the table, all the difference matrices of the new code between any two pairs of codeword matrices are with full rank. Therefore, this code satisfies the rank criterion stated in [8] and achieves full diversity when QPSK constellation is used. On the contrary, there are 1040 difference matrices with rank 2 for Jafarkhani's code. Thus, the SNR-BER curves are dominated by those pairs and the code can achieve only partial diversity order.

The simulation at $3 \mathrm{bits} / \mathrm{s} / \mathrm{Hz}$ using 8 -PSK constellation shows that the SNR-BER curves of our codes are identical to the curve of Jafarkhani's QOSTBC. Only half diversity order is achieved. A similar analysis is done by examining the rank and minimum determinant of the difference matrices between all pairs of codeword matrices in 8-PSK constellation. The results for both our new code and Jafarkhani's code show that although most of the difference matrices are full-rank, 24128 difference matrices are of rank 2 . Thus, it is reasonable that these two codes have exactly the same performance.

Another advantage of our codes is the low decoding computational complexity due to their quasi-orthogonal structure. As shown in [3], QOSTBCs can be decoded pairwisely. For example, the first and second columns of the QOSTBC in (7) are orthogonal to the remaining two columns. To decode the symbol pairs $z_{1}, z_{2}$ and $z_{3}, z_{4}$ separately is an optimal decoding scheme. Thus, there is no need to search all possible symbol sequences at the receiver side. The resulting computational complexity is much less than the computational complexity of the typical ML detection.

The successful experience of constructing a full-diversity design in QPSK constellation implies that to design a fulldiversity code for a specific constellation based on QOST$\mathrm{BCs}$ is feasible. The concept is called a relaxed designing viewpoint. The advantages of the codes based on the relaxed 


\begin{tabular}{|c|c|c|}
\hline No. of rank 2/4 difference matrices & QPSK (total $4^{4} \times 4^{4}-1=32640$ pairs) & 8-PSK (total $8^{4} \times 8^{4}-1=8386560$ pairs) \\
\hline Jafarkhani's code [3] & $1040 / 31600$ & $24128 / 8362432$ \\
\hline New code & $0 / 32640$ & $24128 / 8362432$ \\
\hline
\end{tabular}

TABLE II

NUMBERS OF RANK 2 AND RANK 4 DIFFERENCE MATRICES IN TWO CONSTELLATIONS.

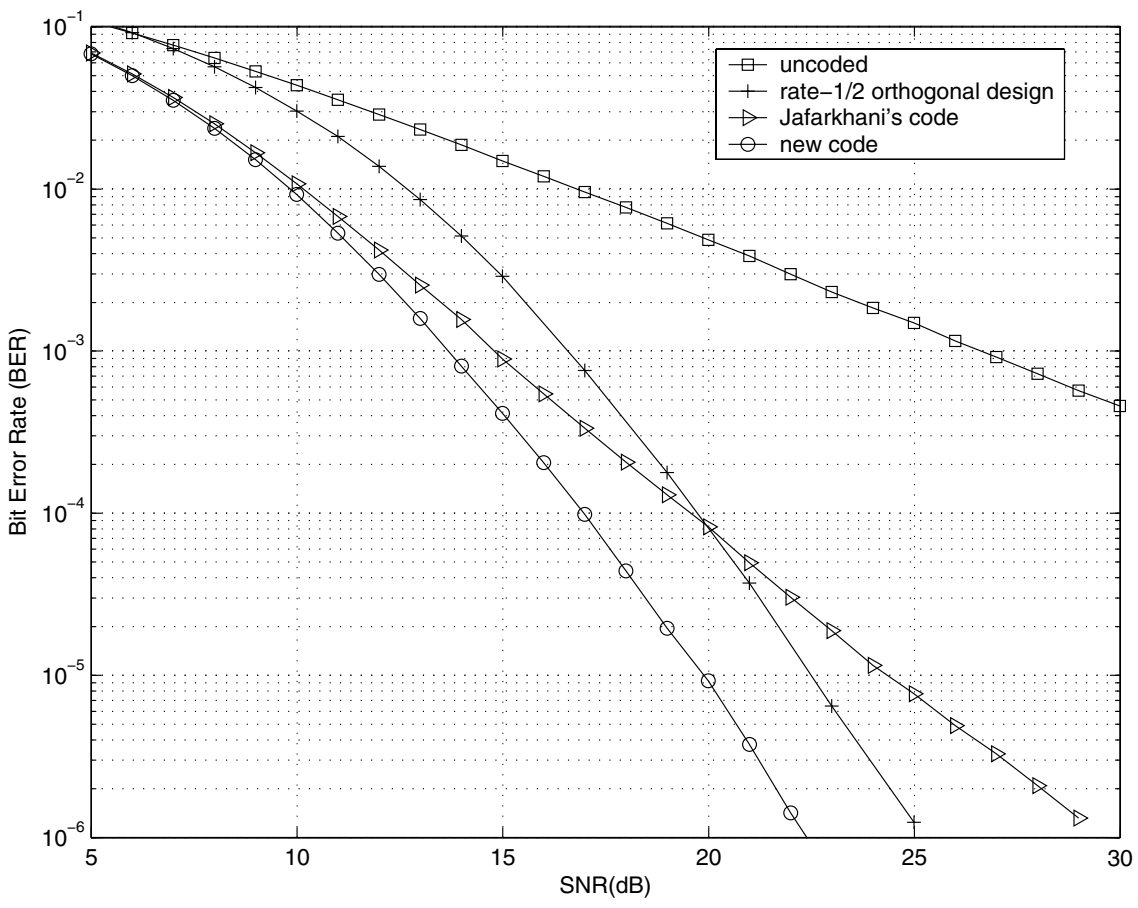

Fig. 1. BER performance for MISO transmission schemes at $2 \mathrm{bits} / \mathrm{s} / \mathrm{Hz}$.

designing viewpoint can be explained in two respects. First, since the codes satisfy the rank criterion in a given constellation, full diversity can be achieved. On the other hand, the ML decoding can be simplified by searching symbol pairs with maximum likelihood because the codes are quasi-orthogonal essentially. In [5] and [6], the quasi-orthogonal codes with constellation rotations can also achieve full diversity. However, an additional constellation has to be used for schemes with rotated constellations. Our codes provide a simpler expression, which achieves full diversity without constellation expansion. And to exploit other full-rate, complex full-diversity designs based on QOSTBCs for all other constellations becomes an interesting open problem.

\section{CONCLUSION}

A novel approach of designing well-performed full-rate quasi-orthogonal space-time block codes on simpler transmission architecture has been proposed. As far as the transmit signal constellations are concerned, our approach makes it possible to construct full-diversity codes for QPSK modulation. We believe the full-rate complex full-diversity designs for any specific constellation can be constructed more efficiently based on the relaxed designing viewpoint in the future.

\section{REFERENCES}

[1] S. M. Alamouti, "A simple transmit diversity technique for wireless communications," IEEE Journal on Selected Areas in Communications, Vol.16, No.8, pp. 1451-1458, October 1998.

[2] H. S. M. Coxeter and W. O. J. Moser, Generators and Relations for Discrete Groups, Springer-Verlag Berlin and Heidelberg, 1980.

[3] H. Jafarkhani, "A quasi-orthogonal space-time block code," IEEE Transactions on Communications, Vol.49, No.1, pp. 1-4, January 2001.

[4] C. B. Papadias and G. J. Foschini, "Capacity-approaching spacetime codes for systems employing four transmitter antennas," IEEE Transactions on Information Theory, Vol.49, No.3, pp. 726-733, March 2003.

[5] N. Sharma and C. B. Papadias, "Improved quasi-orthogonal codes through constellation rotation," IEEE Transactions on Communications, Vol.51, No.3, pp. 332-335, March 2003.

[6] W. Su and X.-G. Xia, "Signal constellations for quasi-orthogonal spacetime block codes with full diversity," IEEE Transactions on Information Theory, Vol.50, No.10, pp. 2331-2347, October 2004.

[7] V. Tarokh, H. Jafarkhani, and A. R. Calderbank, "Space-time block codes from orthogonal designs," IEEE Transactions on Information Theory, Vol.45, No.5, pp. 1456-1467, July 1999.

[8] V. Tarokh, N. Seshadri, and A. R. Calderbank, "Space-time codes for high data rate wireless communication: performance criterion and code construction," IEEE Transactions on Information Theory, Vol.44, No.2, pp. 744-765, March 1998. 